\title{
CONTENT BASED VIDEO RETRIEVAL
}

\author{
B. V. Patel ${ }^{1}$ and B. B. Meshram ${ }^{2}$ \\ ${ }^{1}$ Shah \& Anchor Kutchhi Polytechnic, Mumbai, INDIA \\ patelbveacm.org \\ ${ }^{2}$ Computer Technology Department, VJTI, Mumbai, INDIA \\ bomeshramevjti.org.in
}

\begin{abstract}
Content based video retrieval is an approach for facilitating the searching and browsing of large image collections over World Wide Web. In this approach, video analysis is conducted on low level visual properties extracted from video frame. We believed that in order to create an effective video retrieval system, visual perception must be taken into account. We conjectured that a technique which employs multiple features for indexing and retrieval would be more effective in the discrimination and search tasks of videos. In order to validate this claim, content based indexing and retrieval systems were implemented using color histogram, various texture features and other approaches. Videos were stored in Oracle $9 i$ Database and a user study measured correctness of response.
\end{abstract}

\section{KEYWORDS}

CBVR, Feature Extraction, Indexing

\section{INTRODUCTION}

During recent years, methods have been developed for retrieval of videos based on their visual features. Color, texture, shape, motion and spatial-temporal composition are the most common visual features used in visual similarity match. Realizing that inexpensive storage, ubiquitous broadband Internet access, low cost digital cameras, and nimble video editing tools would result in a flood of unorganized video content; researchers have been developing video search technologies for a number of years. The recent trends in video creation and delivery technology have brought the need for such tools to the forefront. Video retrieval continues to be one of the most exciting and fastest growing research areas in the field of multimedia technology [1].

Despite the sustained efforts in the last years, the paramount challenge remains bridging the semantic gap. By this we mean that low level features are easily measured and computed, but the starting point of the retrieval process is typically the high level query from a human[3]. Translating or converting the question posed by a human to the low level features seen by the computer illustrates the problem in bridging the semantic gap. However, the semantic gap is not merely translating high level features to low level features. The essence of a semantic query is to understand the meaning behind the query. This can involve understanding both the intellectual and emotional sides of the human, not merely the distilled logical portion of the query but also the personal preferences and emotional sub tones of the query and the preferential form of the results. Content-based image retrieval (CBIR), also known as query by image content (QBIC) and content-based visual information retrieval (CBVIR) is the application of computer vision to the video retrieval problem, that is, the problem of searching for video in large databases.

"Content" in this context might refer to colors, shapes, textures, or any other information that can be derived from the image itself. Without the ability to examine video content, searches must rely on metadata such as captions or keywords, which may be laborious or expensive to produce. The 
The International Journal of Multimedia \& Its Applications (IJMA) Vol.4, No.5, October 2012

demand for intelligent processing and analysis of multimedia information has been rapidly growing in recent years[12]. Among them, content-based video retrieval is the most challenging and important problem of practical value. It can help users to retrieve desired video from a large video database efficiently based on the video contents through user interactions. The video retrieval system can be roughly divided into two major components: a module for extracting representative features from video frames and one defining an appropriate similarity model to find similar video frames from database. Many approaches used different kinds of features to represent a video frame, including color histogram, shape information, texture, text analysis. Few approaches integrate features to improve the retrieval performance [8].

Our proposed approach consists of various modules for key frame extraction, indexing, features extraction, similarity search etc. We use a dynamic programming approach to compute the similarity between the feature vectors for the query and feature vectors in the feature database. Proposed Video Storage and Retrieval System, stores and manages a large number of video data and allows users to retrieve videos from the database efficiently. It is interactive web based application which takes video frame from users and retrieve the information from the database. Database consists of various video data like still video frames, audio and video. The retrieval is based on the content of the video object.

Proposed System provides different functionality for two main clients-which are Administrator and user. Administrator is responsible for controlling the entire database including security and adding, updating and deleting videos to and from database. User can only retrieve videos based on submitted query based on content as well on metadata. Rest of the paper is organized as follow: Section two deals with the System Analysis and Design, in section three we discuss system design, section four describes proposed algorithms, we present experimental results in section five and finally section six concludes.

\section{SYSTEM ANALYSIS AND DESIGN}

In this section we present system analysis and design of proposed systems. System analysis model defines user requirements, establishes basis of system design and defines set of validation requirements needed for testing implemented system. System design is the technical kernel of System engineering and is applied regardless of the system process model that is used. Beginning once system requirements have been analyzed and specified, System design is the first of three technical activities - design, code generation, and test - that are required to build and verify the system. Each activity transforms information in a manner that ultimately results in validated proposed System.

\subsection{System Analysis}

At the core of the model lies the data dictionary-a repository that contains descriptions of all data objects of system. Three different diagrams surround the core. The entity relation diagram (ERD) depicts relationships between data objects. The ERD is the notation that is used to conduct the data modeling activity. The attributes of each data object noted in the ERD can be described using a data object description.

The data flow diagram (DFD) serves two purposes- to provide an indication of how data are transformed as they move through the system and to depict the functions and sub functions that transform the data flow.

The DFD provides additional information that is used during the analysis of the information domain and serves as a basis for the modeling of function. A description of each function presented in the DFD is contained in a process specification (PSPEC). The state transition 
The International Journal of Multimedia \& Its Applications (IJMA) Vol.4, No.5, October 2012

diagram (STD) indicates how the system behaves as a consequence of external events. To accomplish this, the STD represents the various modes of behavior (called states) of the system and the manner in which transitions are made from state to state. The STD serves as the basis for behavioral modeling.

\subsection{ER Diagram}

The title is to be written in 20 pt. Garamond font, centred and using the bold and "Small Caps" formats. There should be $24 \mathrm{pt}$. (paragraph) spacing after the last line.

The object/relationship pair is the cornerstone of the data model. These pairs can be represented graphically using the entity/relationship diagram. A set of primary components are identified for the ERD data objects, attributes, relationships, and various type indicators. The primary purpose of the ERD is to represent data objects and their relationships. ER diagram shows the relationship between all the entities in the system. An Entity can be described using a number of attributes. The Relationship shows how all the entities interact with each other. There are two basic entities in proposed system, which are as shown in figure 1.

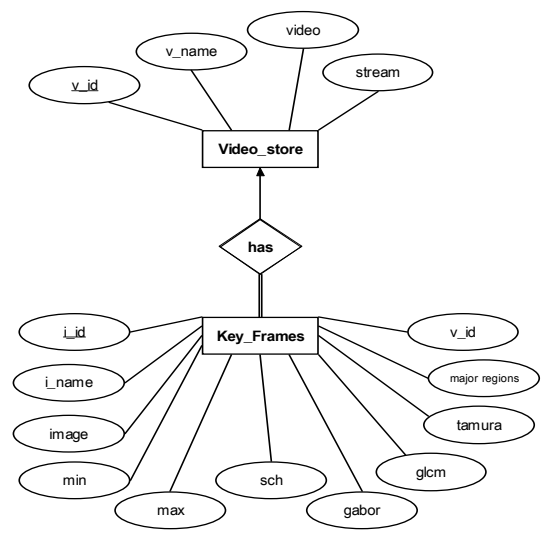

Figure 1. ER Diagram

Attributes: Key_frames(i_id,i_name,image,min,max,sch,gabor,glcm,tamura,majorRegions,v_id) Video_store (v_id,v_name,video,stream)

\subsection{Use Case Diagram}

A use-case is a scenario that describes how System is to be used in a given situation. To create a use-case, the analyst must first identify the different types of people (or devices) that use the system or product. These actors actually represent roles that people (or devices) play as the system operates. An actor is anything that communicates with the system or product and that is external to the system itself. A use case corresponds to a sequence of transaction, in which each transaction is invoked from outside the system (actors) and engages internal objects to interact with one another and with the system surroundings. Use case represents specific flows of events in the system; it is also a graph of actors, a set of use cases enclosed by a systems boundaries communication between actors and the use cases. It is shown in following figure 2 . 
The International Journal of Multimedia \& Its Applications (IJMA) Vol.4, No.5, October 2012

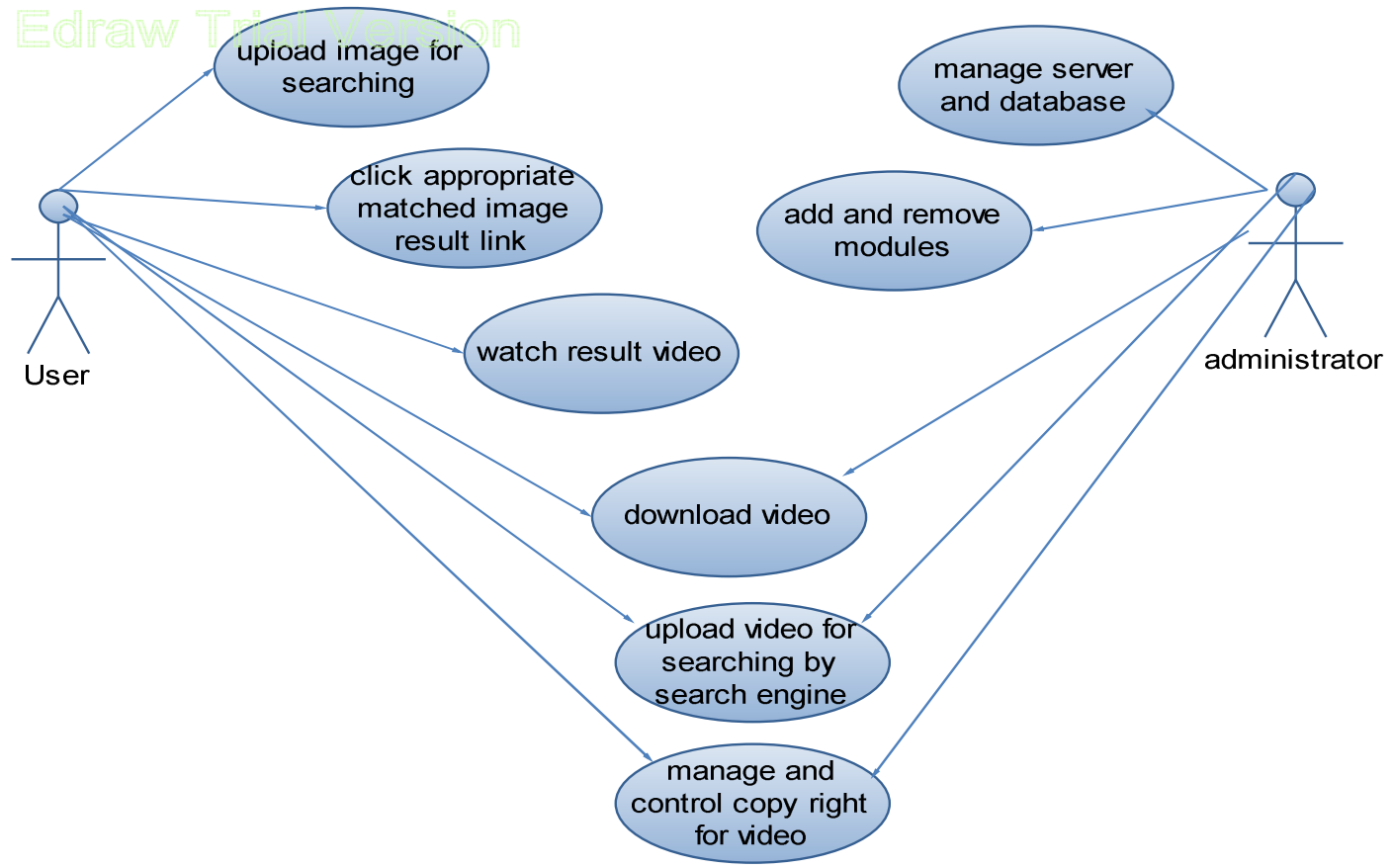

Figure 2. Use case diagram

\subsection{Data Flow Diagram}

As information moves through System, it is modified by a series of transformations. A data flow diagram is a graphical representation that depicts information flow and the transforms that are applied as data move from input to output. The data flow diagram may be used to represent a system or System at any level of abstraction. In fact, DFDs may be partitioned into levels that represent increasing information flow and functional detail. Therefore, the DFD provides a mechanism for functional modeling as well as information flow modeling. Figure 3 demonstrates DFD for proposed systems.

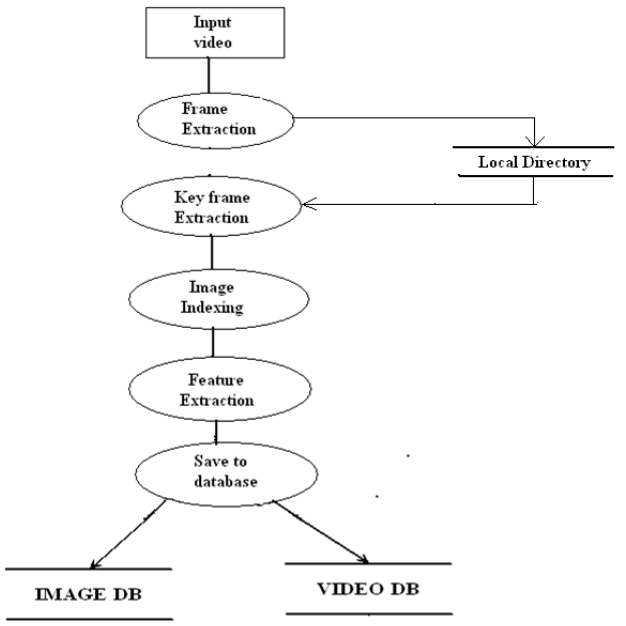

Figure 3. DFD for proposed systems 
The International Journal of Multimedia \& Its Applications (IJMA) Vol.4, No.5, October 2012

\section{SYSTEM DESIGN}

Each of the elements of the analysis model provides information that is necessary to create the design models required for a complete specification of design. System requirements, manifested by the data, functional, and behavioral models feed the design task. Using one of a number of design methods, the design task produces a data design, an architectural design, an interface design, and a component design.

\subsection{Block Diagram of Proposed System}

The System contains two main modules. The Administrator is responsible for addition, deletion and modification of the multimedia objects. User can use only the search engine to search image, audio or video as shown in figure 4 .

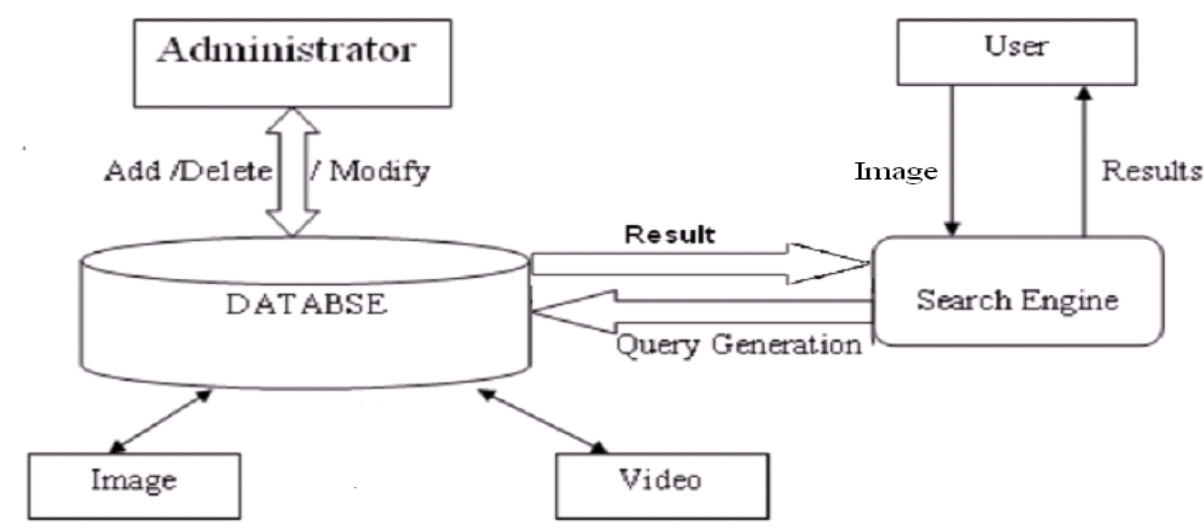

Figure 4. System Block Diagram

\subsection{Component Diagram}

It models the physical components in design the way of looking at the components is the packages. Apackage is used to show how we can group together classes, which in essence are smaller scale components .

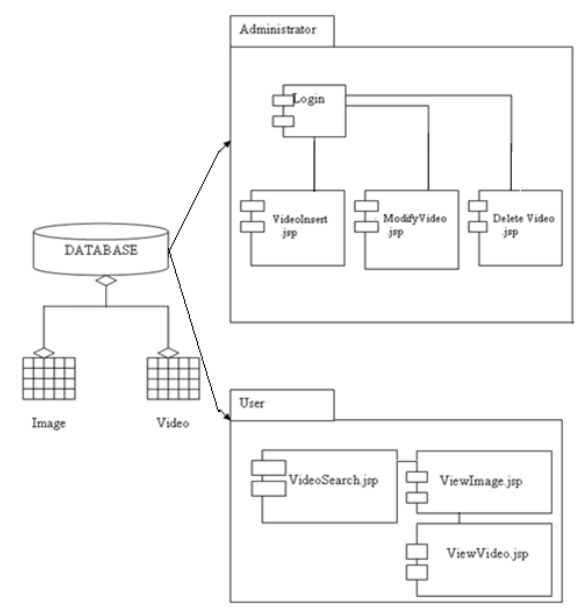

Figure 5. Component diagram 
The International Journal of Multimedia \& Its Applications (IJMA) Vol.4, No.5, October 2012

Here administrator manages login, addition, modification and deletion of video from database and user searches video based on index frame and views similar videos as shown in figure 5 .

\subsection{Deployment Diagram}

Deployment Diagram shows the configuration of runtime processing elements and the software components, processes and objects that live in them. It is shown in figure 6. A software component instance represents runtime manifestations of code units. In most cases, component diagrams are used in the conjunction with deployment diagram to show how physical modules of code are distributed on various hardware platforms. In many cases, component and deployment diagrams can be combined. A deployment diagram is graph of nodes connected by communication association. Nodes may contain a component instance, which means that component lives or runs at that mode. Components may contain objects; this indicates that the object is a part of component. Components are connected to other components by dashed arrow dependencies, usually through interfaces, which indicate one component uses service of another. Each node or processing element in the system represented by a three dimensional box. Connection between the node (or platforms) themselves are shown by solid lines.

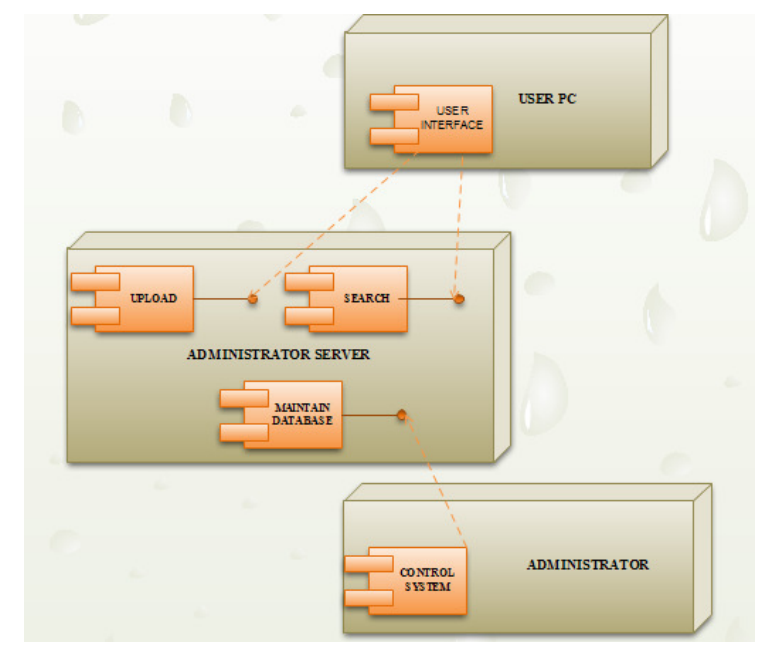

Figure 6. Deployment Diagram

\subsection{Database Design}

Database design is used to define and then specifies the structure of business objects used in the client/server system. The analysis required to identify business objects is accomplished using business process engineering methods. Conventional analysis modeling notation such as the ERD, can be used to define business objects, but a database repository should be established in order to capture the additional information that cannot be fully documented using a graphic notation such as an ERD.

In this repository, a business object is defined as information that is visible to the purchasers and users of the system, not its developers. This information, implemented using a relational database, can be maintained in a design repository. Following are the database tables used for implementing proposed systems on which queries can be performed.

Video_store(v_id, v_name, video,stream)

Key_frames(v_id,i_name,image,min,max,sch,gabor,glcm,tamura,majorRegions,v_id) 
The International Journal of Multimedia \& Its Applications (IJMA) Vol.4, No.5, October 2012

Where v_id - video id,

v_name - name of video file,

video - video file,

stream - stream of keyframes,

i_id - video frame id,

min-max - indexing range,

sch - simple colour histogram,

gabor, glcm, tamura - feature texture string,

majorRegion - no. of max regions

Using following Oracle query Video_store table is created for indexing and retrieval of video.

CREATE TABLE "VIDEO_STORE"

( "V_ID" NUMBER NOT NULL ENABLE,

"V_NAME" VARCHAR2(60),

"VIDEO" ORD_ Video,

"STREAM" BLOB,

"DOSTORE" DATE,

)

PRIMARY KEY ("V_ID") ENABLE

Using following Oracle query KEY_FRAMES table is created for storing key frames and their features.

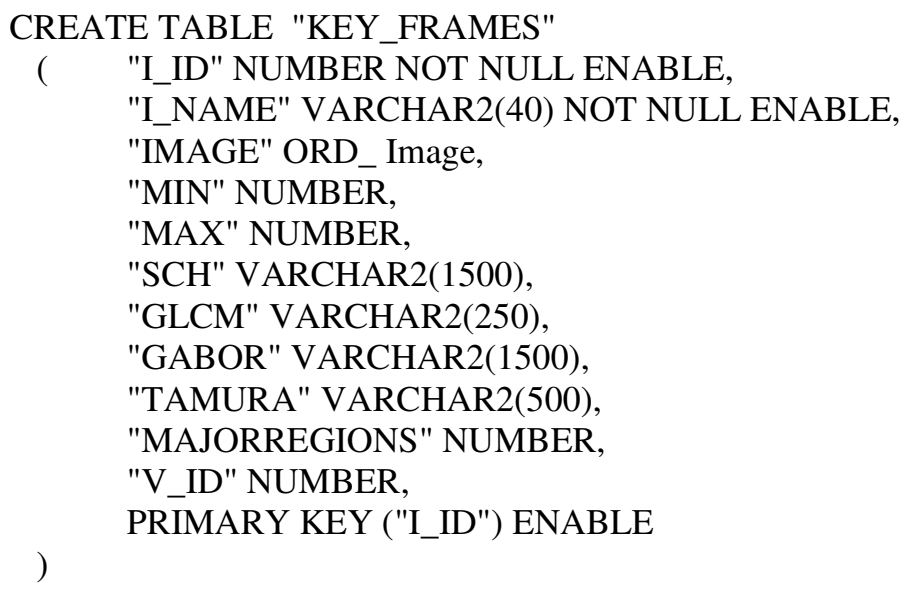

\section{Algorithms Proposed ANd USed}

\subsection{Key frame extraction Algorithm}

It works on group of frames extracted from a video. It takes a list of files or frames in order in which they will be extracted. It is based on predefined threshold that specify whether 2 video frame are similar. It $\mathrm{s}$ main function is to choose smaller number of video representative key frames.

Starts from $1^{\text {st }}$ frame from sorted list of files.

If consecutive frames are within threshold, then two frames are similar.

Repeat process till frames are similar, delete all similar frames $\&$ take $1^{\text {st }}$ as key -frame.

Start with next frame which is outside threshold \& repeat the steps i to a for all frames of video .

Input - Frames of video extracted by video to jpeg converter. 
The International Journal of Multimedia \& Its Applications (IJMA) Vol.4, No.5, October 2012

Output - Key frames with less similarity \& representing video.

\section{Pseudo code for key frame extraction}

1] Let i \& j be integer variables, ri1 \& ri2 be RenderedImage objects.

2] Get all Jpeg files in files array.

3] Initialise length to length of files array.

4] Do

a] $\mathrm{i}=0$

b] while $\mathrm{i}$ is not equal to length

i] Create RenderedImage ril object that hold rescaled IVersion of image file $i$.

ii] Do

a] $\mathrm{j}=\mathrm{i}+1$

b] while $\mathrm{j}$ is not equal to length image file $\mathrm{j}$.

c] Create RenderedImage ri2 object that holds rescaled IVersion of

c] $i=i+1$

d] dist $=$ difference between ri1 \& ri2.

e] if(dist $>800.0)$

else

$\{\mathrm{i}=\mathrm{j}-1$; break; $\}$

delete file $\mathrm{j}$.

5] End

$$
\text { f] } j=j+1
$$

\subsection{Histogram Based Range Finder Indexing Algorithm}

We implemented tree based indexing algorithm consisting of grouping frames into pixel ranges such as 0-255 on first level, 0-127 or 128-255 on second level so on third, fourth level. Algorithm level by level calculates min - max range finally if when thresholding criteria is not satisfied, frame is grouped onto previous level into min -max range. Every frame is part of first level as it satisfies thresholding criteria.

1.Calculate histogram of image.

2.Calculate min-max range by grouping pixel count.

3. Find if thresholding criteria are satisfied.

4. Go to next lower level \& repeat step from 2 to 4 .

5.If criteria are not satisfied, image is grouped on previous level where min - max range is satisfied as shown in figure 7.

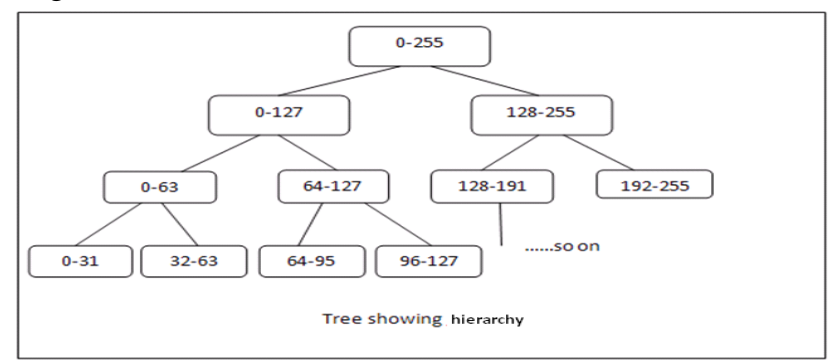

Figure 7. Indexing tree

\section{Indexing:}

Input : Video frame after choosing key frames as image. 
The International Journal of Multimedia \& Its Applications (IJMA) Vol.4, No.5, October 2012

Output : $\min -\max$ range that groups image in particular group.

Pseudo code for indexing process :

Variables min \& max store range for indexing process.

1] Let $\min =0, \max =255$;

2] Let long sum $=0$;

3] Let double result $=0$;

4] //1st block test

A] $\mathrm{i}=\min$

$\mathrm{B}$ ] while $\mathrm{i}$ is not equal to max

i] $\operatorname{sum}=$ sum + histogram $[\mathrm{i}]$

C] result $=$ sum $/ 900.0$;

D] if(result $>55.0)$

$\{\min =0 ; \max =127 ;\}$

else

$\{\min =128 ; \max =255 ;\}$

E] $\mathrm{i}=\mathrm{i}+1$

5] //2nd block test

sum $=0$, result $=0$;

6] if $(\min ==0 \& \& \max ==127)$

\{

A] $\mathrm{i}=0$

B] while $\mathrm{i}$ is not equal to 63

i] sum = sum + histogram [i]

C] result $=$ sum $/ 900.0$;

D] if(result $>60.0)$

$\{\min =0 ; \max =63 ;\}$

else

\{

I] sum $=0$, result $=0$;

II] for (int $\mathrm{i}=64 ; \mathrm{i}<127$; i++)

a] sum = sum + histogram $[\mathrm{i}]$;

III] result $=$ sum $/ 900.0$;

IV] if(result $>60.0)$

\}

$\{\min =64 ; \max =127 ;\}$

\}

7] $\operatorname{sum}=0$, result $=0$;

8] if $(\min ==128 \& \& \max ==255)$

\{

A] for (int $\mathrm{i}=128 ; \mathrm{i}<191 ; \mathrm{i}++$ )

i] $\operatorname{sum}=$ sum + histogram $[i]$;

B] result $=$ sum $/ 900.0$;

C] if(result $>60.0)$

$\{\min =128 ; \max =191 ;\}$

else

\{

I] sum $=0$, result $=0$;

II] for (int $\mathrm{i}=192 ; \mathrm{i}<255$; $\mathrm{i}++$ )

a] $\operatorname{sum}=$ sum + histogram $[\mathrm{i}]$;

III] result = sum/900.0;

IV] if(result $>60.0$ )

$\{\min =192 ; \max =255 ;\}$

\}

\} 
The International Journal of Multimedia \& Its Applications (IJMA) Vol.4, No.5, October 2012

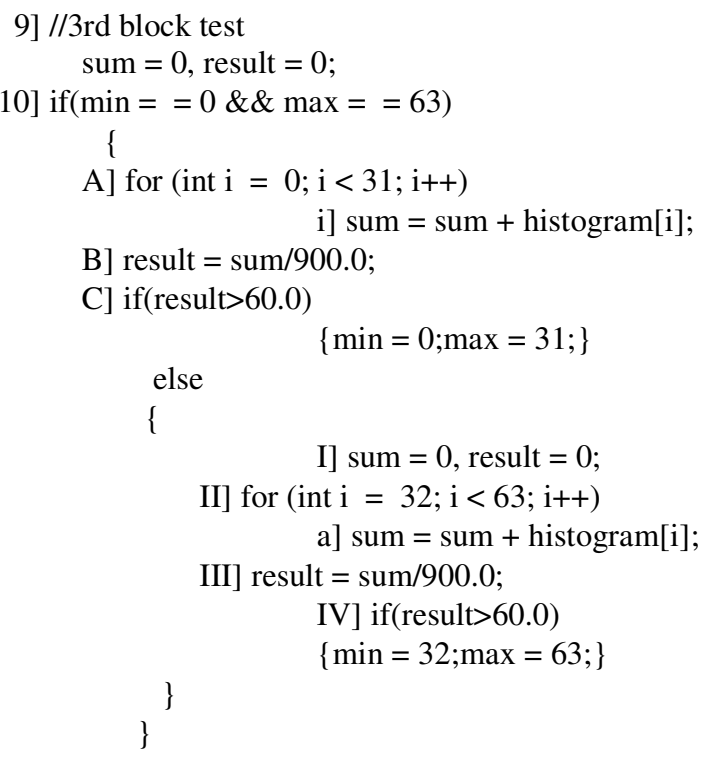

11] sum $=0$, result $=0$;

12] if $(\min ==64 \& \& \max ==127)$

\{

A] for (int $\mathrm{i}=64 ; \mathrm{i}<95 ; \mathrm{i}++$ )

i] $\operatorname{sum}=$ sum + histogram $[\mathrm{i}]$;

B] result $=$ sum $/ 900.0$;

C] if(result $>60.0)$

$\{\min =64 ; \max =95 ;\}$

else

I] $\operatorname{sum}=0$, result $=0$;

II] for (int i $=96 ; \mathrm{i}<127 ; \mathrm{i}++$ )

a]sum $=$ sum + histogram $[i]$;

III] result $=$ sum $/ 900.0$;

IV] if(result $>60.0$ )

$\{\min =96 ; \max =127 ;\}$

\}

\}

13] sum $=0$, result $=0$;

14] if $(\min ==128 \& \& \max ==191)$

\{

A] for (int $\mathrm{i}=128 ; \mathrm{i}<159 ; \mathrm{i}++$ )

i] sum = sum + histogram $[\mathrm{i}]$;

B] result $=$ sum $/ 900.0$;

C] if(result $>60.0)$

$\{\min =128 ; \max =159 ;\}$

else

I] $\operatorname{sum}=0$, result $=0$;

II] for (int $\mathrm{i}=160 ; \mathrm{i}<191 ; \mathrm{i}++$ )

a] sum = sum + histogram $[\mathrm{i}]$;

III] result = sum/900.0;

IV] if(result $>60.0)$

$\{\min =160 ; \max =191 ;\}$ 
The International Journal of Multimedia \& Its Applications (IJMA) Vol.4, No.5, October 2012

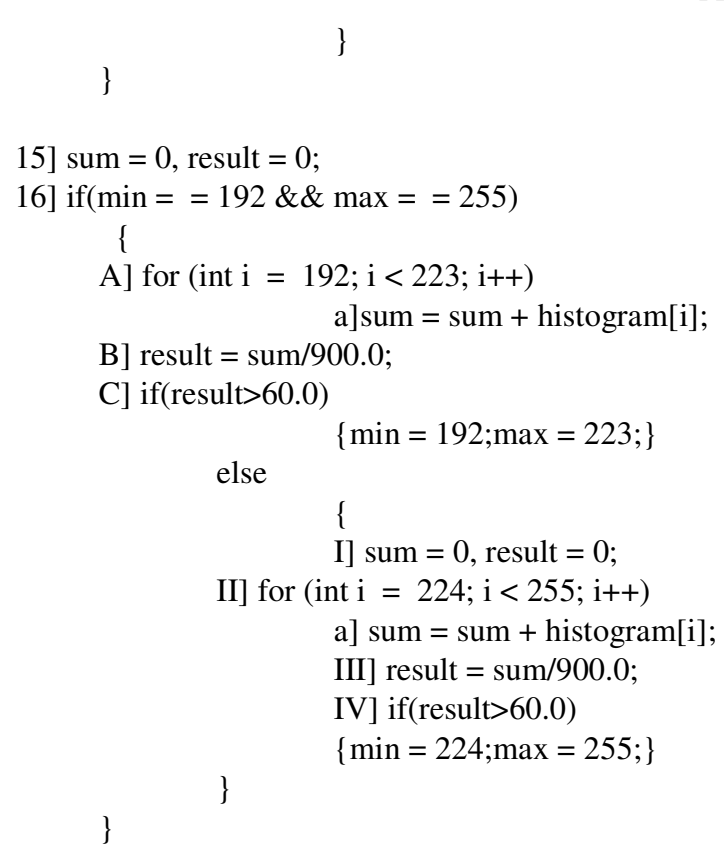

\subsection{GLCM Texture Feature Extraction}

The Gray Level Co-occurrence Matrix1 (GLCM) and associated texture feature calculations are image analysis techniques. Given an image composed of pixels each with an intensity (a specific gray level), the GLCM is a tabulation of how often different combinations of gray levels co-occur in an image or image section.

Input : image as PlanarImage object

Output : string containing feature values.

Pseudo code for GLCM texture:

public GLCM_Texture(PlanarImage im, boolean preprocess):

1] If(preprocessor is true) input = preprocess the image in im;

2] otherwise input $=\mathrm{im}$;

3] create a data structure Raster for input image

4] initialize $\mathrm{k}=0, \mathrm{~h}=\mathrm{O}, \mathrm{w}=0$;

i] while $h$ is not equal to height of image

a] while $\mathrm{i}$ is not equal to width of image ;

A] Initialise pixels[w][h] with value for pixel in image.

B] Initialise pixel[k++] with value for pixel in image.

private PlanarImage preprocess(PlanarImage input)

\{

1] if (input has IndexColorModel)

A] Retrieve the IndexColorModel of image;

B] Cache the number of elements in each band of the colormap in mapSize integer variable.

C] Allocate an array for the lookup table data i.e. lutData $=$ new byte[3][mapSize];

D] Load the lookup table data from the IndexColorModel.

i] Get Red in lutData[0] from IndexColorModel object ;

ii] Get Green in lutData[1] from IndexColorModel object ;

iii] Get Blue in lutData[2] from IndexColorModel object ; 
The International Journal of Multimedia \& Its Applications (IJMA) Vol.4, No.5, October 2012

E] Create the lookup table object i.e. LookupTableJAI using lutData array;

F] Replace the original image i.e. input with the 3-band RGB image using lut object.

2] if ( NumBands in input $>1$ )

A] Let matrix $=\{\{0.114,0.587,0.299,0\}\}$;

B] Replace the original image i.e. input with bandcombineb using matrix;

3]return input;

public void calcTexture()

1] Let $\mathrm{a}=0$ be integer variables;

2] Let $b=0, y=0, x=0$ be integer variables;

3] Let offset and $i$ be integer variables;

4] Let glcm be a 2-D array of 257 by 257 ;

5] while y is not equal to height

i] offset $=y^{*}$ width;

6] while $x$ is not equal to width

i] $\mathrm{i}=$ offset $+\mathrm{x}$;

ii] $\mathrm{a}=0 \mathrm{xff} \& \operatorname{pixel}[\mathrm{i}]$

iii] $\mathrm{b}=0 \mathrm{xff} \& \operatorname{pixels}[\mathrm{x}+$ step $][\mathrm{y}]$;

iv] glcm [a][b] $+=1$;

v] glcm [b][a] $+=1$;

vi] pixelCounter $+=2$;

7] while a is not equal to 257

i] while $b$ is not equal to 257

a] $\operatorname{glcm}[\mathrm{a}][\mathrm{b}]=\operatorname{glcm}[\mathrm{a}][\mathrm{b}] /$ pixelCounter;

8] while a is not equal to 257

i] while $b$ is not equal to 257

9] Initialize double $p x=0$;

a] asm $=\operatorname{asm}+\left(\operatorname{glcm}[\mathrm{a}][\mathrm{b}]^{*} \mathrm{glcm}[\mathrm{a}][\mathrm{b}]\right)$;

10] Initialize double $p y=0$;

11] Initialize double mean $x=0.0$;

12] Initialize double meany $=0.0$;

13] Initialize double stdev $x=0.0$;

14] Initialize double stdevy $=0.0$;

15] while a is not equal to 257

i] while $b$ is not equal to 257

a] $p x=p x+a^{*} g l c m[a][b]$;

b] $p y=p y+b^{*}$ glcm [a][b];

16] while a is not equal to 257

i] while $b$ is not equal to 257

a] $\operatorname{stdevx}=\operatorname{stdevx}+(a-p x) *(a-p x] * g l c m[a][b] ;$

b] stdevy $=$ stdevy+(b-py)*(b-py)*glcm [a][b];

17] while a is not equal to 257

i] while b is not equal to 257

a] correlation $=$ correlation $+(a-p x) *(b-p y) * g l c m[a][b] /(\operatorname{stdevx} * \operatorname{stdevy}) ;$

18] while a is not equal to 257

i] while $b$ is not equal to 257

a] IDM=IDM+glcm[a][b]/(1+(a-b)*(a-b))

19] while a is not equal to 257

i] while b is not equal to 257

$$
\begin{aligned}
& \text { a] if }(\operatorname{glcm}[\mathrm{a}][\mathrm{b}]==0)\{\} \\
& \text { b] else } \\
& \text { entropy }=\text { entropy }-\operatorname{glcm}[\mathrm{a}][\mathrm{b}] * \log (\operatorname{glcm}[\mathrm{a}][\mathrm{b}])
\end{aligned}
$$

public String getStringRepresentation()

1] Build new object StringBuilder sb; 
The International Journal of Multimedia \& Its Applications (IJMA) Vol.4, No.5, October 2012

2] Append the string GLCM texture to sb;

3] Append pixelCounter to sb;

4] Append asm to sb;

5] Append contrast to sb;

6] Append correlation to sb;

7] Append IDM to sb;

8] Append entropy to sb;

9] Return string format of sb;

\subsection{Gabor Texture Feature}

Gabor Feature is a linear filter used for edge detection. Frequency and orientation representations of Gabor filters are similar to those of the human visual system, and they have been found to be particularly appropriate for texture representation and discrimination.

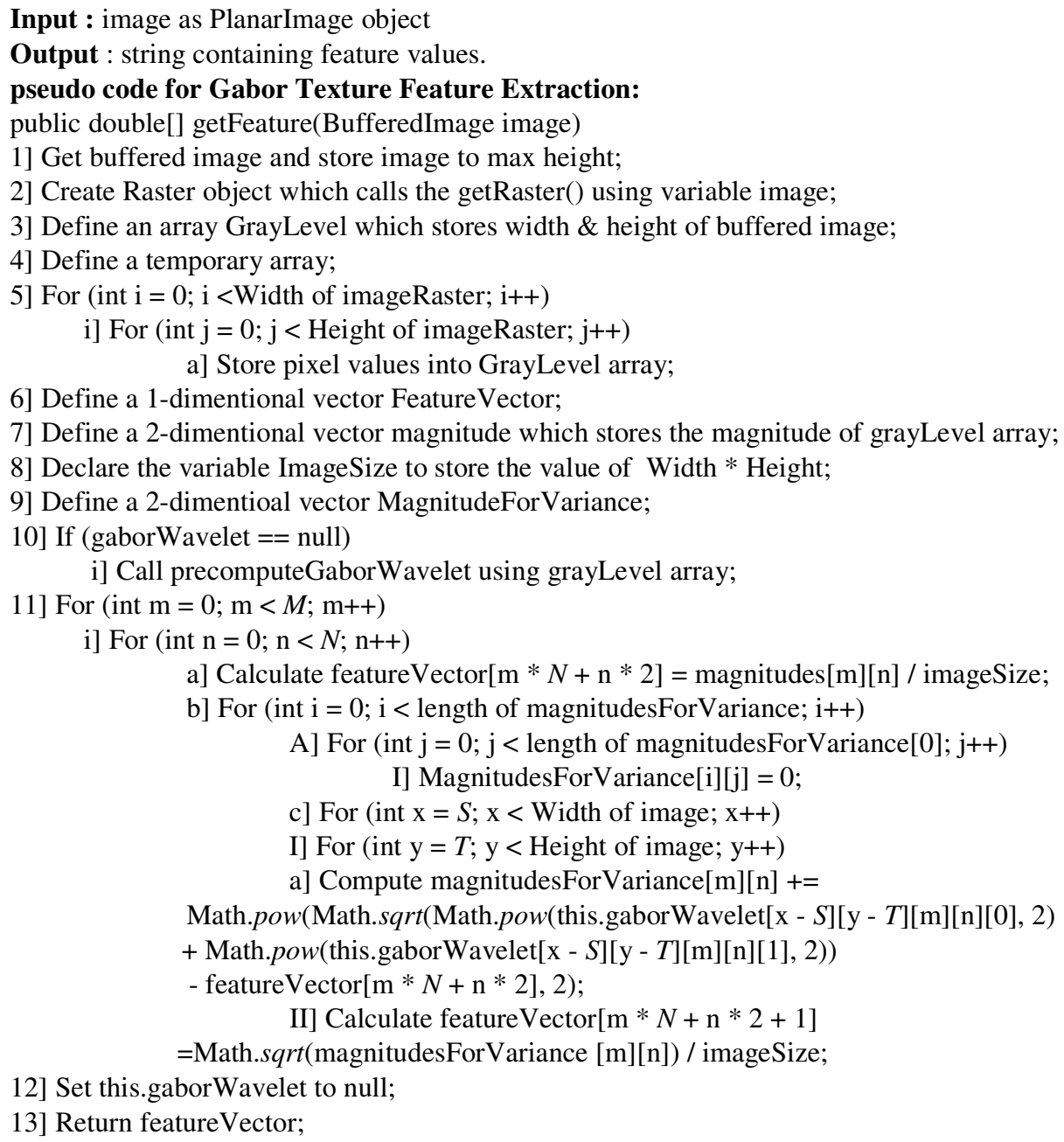

\subsection{Color histogram Extraction}

The color space of frame is quantizes into a finite number of discrete levels. Each of this level becomes bin in the histogram. The color histogram is then computed by counting the number of 
The International Journal of Multimedia \& Its Applications (IJMA) Vol.4, No.5, October 2012

theses discrete levels. Color Histogram(h) is then computed with the color information like $h_{r}(i)$, $h_{g}(i), h_{b}(i)$ to represent the color domain.

Input : image as BufferedImage object

Output : string containing histogram values.

pseudo code for color histogram :

public void extract(BufferedImage image)

1] Let pixel be 2-D array of size width \& height.

2] Create a data structure Raster for input image.

3] $x=0, y=0$ \& while $x$ is not equal to width of image

A] while y is not equal to height of image

i] $\operatorname{pixel}[\mathrm{x}][\mathrm{y}]=$ corresponding value in raster.

ii] if(histogramType != HistogramType.HMMD) histogram[quant(pixel)]++.

public String getStringRepresentation()

1] Create a StringBuilder object.

2] Append histogramType to sb.

3] Append ' ' to sb.

4] Append length of histogram to sb.

5] Append ' ' to sb.

6] for (int $\mathrm{i}=0$; $\mathrm{i}<$ length of histogram. $\mathrm{i}++$ )

A] Append histogram[i] to sb.

B] Append ' ' to sb.

7] Return string format of $s b$.

\subsection{Superficial (native) similarity Algorithm}

This approach is to extract image signature with 25 representative pixels, each in R, G, B. For each of 25 locations over image take $5 * 5$ matrix \& find mean pixel value for matrix. Using this process vector array with 25 mean values for image signature is created.

Input : images as BufferedImage object

Output : difference between image signatures.

pseudo code for Superficial (naive) similarity descriptor:

private RenderedImage rescale(RenderedImage i)

1] float scaleW $=300$, scale $=300$;

// Scales the original image

2] Create ParameterBlock $\mathrm{pb}=$ new ParameterBlock();

3] Adding input image to $\mathrm{pb}$

5] Adding height required to $\mathrm{pb}$

6] Adding filter InterpolationNearest for scaling to $\mathrm{pb}$

7] Return scaled image

private Color averageAround(RenderedImage i, double px, double py)

1] Create RandomIter object named as iterator.

2] let pixels $\&$ accum be 1-D arrays

3] Let sampleSize $=15$, numPixels $=0$.

4] for (double $\mathrm{x}=\mathrm{px} *$ baseSize - sampleSize; $\mathrm{x}<\mathrm{px} *$ baseSize + sampleSize; $\mathrm{x}++$ )

I] for (double y = py * baseSize - sampleSize; y< py * baseSize + sampleSize; $\mathrm{y}++$ )

A] Get pixel value at $x, y$ location.

B] accum $[0]+=\operatorname{pixel}[0]$.

C] accum[1] $+=\operatorname{pixel}[1]$.

D] accum[2] $+=\operatorname{pixel}[2]$.

E] numPixels++. 
The International Journal of Multimedia \& Its Applications (IJMA) Vol.4, No.5, October 2012

5] Average the accumulated values.

accum $[0] /=$ numPixels, accum[1] /= numPixels, accum[2] /= numPixels.

6] return Color object with accum[0], accum[1], accum[2] as RGB values.

public String getStringRepresentation()

1] StringBuilder $\mathrm{sb}=$ new StringBuilder(25*5);

2] append("NaiveVector") to $s b$

3] append(' ') to $\mathrm{sb}$

4] for (int $i=0 ; i<$ length of signature; $i++$ )

I] for (int $j=0 ; j<$ length of signature $[i] ; j++$ )

A] append(signature[i][j]) to $s b$

B] append(' ') to sb

5] Return string format of sb;

\subsection{Autocorrelogram for color}

A color correlogram expresses how the spatial correlation of pairs of colors changes with distance. A color histogram captures only the color distribution in an image and does not include any spatial correlation information.

Input : images as BufferedImage object

Output : string containing correlogram values.

pseudo code for autocorrelogram :

public void extract (BufferedImage bi)

1] Get Raster $r$ from bi object's raster producing method;

2] Create array histogram = new int[256];

3 ] for (int $\mathrm{i}=0 ; \mathrm{i}<$ length of histogram; $i++$ )

a] Initialise histogram $[i]=0$;

4] Create array quantPixels = new int[width of raster][height of raster]

5] quantize colors for each pixel (done in HSV color space):

Create array pixel $=$ new int[3], hsv $=$ new int[3];

6] for (int $\mathrm{x}=0 ; \mathrm{x}<$ width of raster ; $\mathrm{x}++$ )

a] for (int $y=0 ; y<$ height of raster $; y++$ )

I] converting to HSV: convertRgbToHsv using pixel from raster r, hsv array

II] quantize the actual pixel: quantPixels[x][y] = quantize hsv

III] for normalization: histogram[quantPixels[x][y]]++

7] Create array correlogram $=$ new float[256] [maxDistance];

8] for (int i1 = 0; i1 <length of correlogram; i1++)

a] for (int $\mathrm{j}=0 ; \mathrm{j}<$ length of correlogram[i1]; $\mathrm{j}++$ )

I] correlogram $[\mathrm{i} 1][\mathrm{j}]=0$;

9] Create array tmpCorrelogram $=$ new int [maxDistance];

10] for (int $x=0 ; x<$ Width of rster $r ; x++$ )

a] for (int $y=0 ; y<$ Height of rster $r ; y++$ )

I] Create variable color $=$ quantPixels $[\mathrm{x}][\mathrm{y}]$;

II] getNumPixelsInNeighbourhood(x, y, quantPixels, tmpCorrelogram)

III] for (int $\mathrm{i}=0 ; \mathrm{i}<\operatorname{maxDistance} ; \mathrm{i}++$ )

A] correlogram[color][i] $+=$ tmpCorrelogram $[i]$;

11]Create array $\max =$ new float $[$ maxDistance] $\&$ initialise with 0

12]for (int $\mathrm{c}=0$; $\mathrm{c}<$ numBins; $\mathrm{c}++$ )

a]for (int $\mathrm{i}=0 ; \mathrm{i}<\operatorname{maxDistance} ; \mathrm{i}++$ )

I] $\max [\mathrm{i}]=$ Maximum betweencorrelogram[c][i] \& $\max [\mathrm{i}]$

13] for (int $\mathrm{c}=0$; $\mathrm{c}<$ numBins; $\mathrm{c}++$ ) \{

a] for (int $\mathrm{i}=0 ; \mathrm{i}<\operatorname{maxDistance} ; \mathrm{i}++$ )

I] $\operatorname{correlogram}[\mathrm{c}][\mathrm{i}]=\operatorname{correlogram}[\mathrm{c}][\mathrm{i}] / \max [\mathrm{i}]$; 
The International Journal of Multimedia \& Its Applications (IJMA) Vol.4, No.5, October 2012

\subsection{Simple Region Growing}

Region growing is a simple region-based image segmentation method. It is also classified as a pixel-based image segmentation method. It is a classic stack-based region growing algorithm:

Find a pixel which is not labeled. Label it and store its coordinates on a stack.

While there are pixels on the stack, do:

Get a pixel from the stack (the pixel being considered).

Check its neighbors to see if they are unlabeled and close to the considered pixel; if are, label them and store them on the stack.

Repeat all steps until there are no more pixels on the image.

Input : images as BufferedImage object

Output : number of regions, number of holes , number of major regions.

pseudo code for Simple Region Growing :

private PlanarImage preprocess(PlanarImage input)

1] if (input has IndexColorModel)

A] Retrieve the IndexColorModel in IndexColorModel icm object;

B] Cache the number of elements in each band of the colormap in mapSize integer

variable.

C] Allocate an array for the lookup table data i.e.byte[][] lutData = new

byte[3][mapSize];

D] Load the lookup table data from the IndexColorModel.

i] Get Red in lutData[0] from icm object ;

ii] Get Green in lutData[1] from icm object ;

iii] Get Blue in lutData[2] from icm object ;

E] Create the lookup table object i.e. LookupTableJAI lut using lutData array;

F] Replace the original image i.e. input with the 3-band RGB image using lut object.

2] if ( NumBands in input $>1$ )

A] Let matrix $=\{\{0.114,0.587,0.299,0\}\}$;

B] create a new ParameterBlock;

C] AddSource input image to pb;

D] Add matrix to pb;

E] Replace the original image i.e. input with bandcombineb;

3] Should we binarize it?

A] create a new ParameterBlock;

B] Add source input image to pb;

C] Add sampling parameter to $\mathrm{pb}$

D] Add new int[]\{256\} to $\mathrm{pb}$;

E] Add new double[] $\{0\}$ to $\mathrm{pb}$;

F] Add new double[] $\{256$ to $\mathrm{pb}$;

G] Calculate the histogram of the image and its Fuzziness Threshold.

Create PlanarImage dummyImage to hold histogram ;

$\mathrm{H}]$ Histogram $\mathrm{h}=$ Property of histogram from dummyImage;

J] double[] thresholds = h.getMinFuzzinessThreshold();

$\mathrm{K}$ ] create a new ParameterBlock;

L] AddSource input image to pb;

M] Add thresholds[0] to pb;

N] Replace the original image i.e. input with bynarized;

4] Let kernelMatrix $=\{0,0,0,0,0$, 
The International Journal of Multimedia \& Its Applications (IJMA) Vol.4, No.5, October 2012

$$
\begin{aligned}
& 0,1,1,1,0 \\
& 0,1,1,1,0 \\
& 0,1,1,1,0 \\
& 0,0,0,0,0\}
\end{aligned}
$$

5] Create the kernel object using the array kernelMatrix.

6] create a new ParameterBlock;

7] AddSource input image to $\mathrm{p}$;

8] Add kernel to p;

9] Replace the original image i.e. input with dilated;

10] create a new ParameterBlock;

11] AddSource input image to p;

12] Add kernel to $p$;

13] Replace the original image i.e. input with erode;

14] create a new ParameterBlock;

15] AddSource input image to $\mathrm{p}$;

16] Add kernel to p;

17] Replace the original image i.e. input with erode;

19] create a new ParameterBlock;

20] AddSource input image to $\mathrm{p}$;

21] Add kernel to $p$;

22] Replace the original image i.e. input with dilated;

23] return input image object;

public void run()

1] numberOfRegions $=0$; numhole $=0$;

2] Create a Stack of Point objects with mustDo as name;

3] for(int $h=0 ; h<h e i g h t ; h++)$

A] for(int $w=0 ; w<$ width; $w++$ )

I] position++;

II] if (labels[w] $[\mathrm{h}]<0)$

a] if(pixels $[\mathrm{w}][\mathrm{h}]==0$ )

i] numhole++;

b] numberOfRegions++;

c] Add a new Point(w,h) to mustDo;

d] labels $[\mathrm{w}][\mathrm{h}]=$ numberOfRegions;

e] put(numberOfRegions, 1) into count;

III] while(mustDo.size ()$>0$ )

a] Point thisPoint $=$ mustDo.get $(0)$; mustDo.remove $(0)$;

b]for(int th $=-1 ;$ th $<=1 ;$ th ++ )

i] for(int tw $=-1 ; \mathrm{tw}<=1 ; \mathrm{tw}++)$

1)int $\mathrm{rx}=$ thisPoint $\mathrm{x}+\mathrm{tw}$;

2)int ry = thisPoint. $y+$ th;

3)if $((\mathrm{rx}<0)\|(\mathrm{ry}<0)\|(\mathrm{ry}>=$ height $) \|(\mathrm{rx}>=$ width $))$ continue; 4)if (labels[rx][ry] $<0$ )

A)if (pixels[rx][ry] == pixels[thisPoint.x][thisPoint.y])

i)mustDo.add(new Point(rx,ry));

ii)labels[rx][ry] = numberOfRegions;

iii)count.put(numberOfRegions, count.get(numberOfRegions)+1);

4] position $=$ width $*$ height;

\section{EXPERIMENTAL RESULTS}

Java programming language is used for implementing proposed system. Ecllipse, Jcreator, Apache Tomcat server, Oracle 9i are used for the development of the system. Proposed systems is 
The International Journal of Multimedia \& Its Applications (IJMA) Vol.4, No.5, October 2012

implemented for the java virtual machine enabled windows based pc's with internet connection. Sample videos and frames were downloaded from www.archive.org. We have considered different categories of images like e-learning, sports, cartoon, movies, etc. Table II presents the average precision values at the top 20,30,50, and 100 retrieved video based on various features. We chose these recall points comes from the fact that for retrieval, the system is able to present 25 or 30 thumbnails per page (or screen), and browsing a set of 60 or 100 key frames is not too painful for users (for instance, the well-known Google web retrieval system (http://www.google.com) presents by default 20 query results for images, and 10 query results for text).

Table 1. PRECISION AT 20, 30, 50 AND 100 DOCUMENTS.

\begin{tabular}{|l|c|l|c|c|c|c|c|}
\hline & GLCM & Gabor & $\begin{array}{c}\text { Tamu } \\
\text { ra }\end{array}$ & $\begin{array}{c}\text { Histog } \\
\text { ram }\end{array}$ & $\begin{array}{c}\text { Autocor } \\
\text { relogra } \\
\mathbf{m}\end{array}$ & $\begin{array}{c}\text { Simple } \\
\text { Region } \\
\text { Grawin } \\
\text { g }\end{array}$ & $\begin{array}{c}\text { Combin } \\
\text { ed }\end{array}$ \\
\hline $\begin{array}{l}\text { Avg. } \\
\text { prec.at 20 } \\
\text { frames }\end{array}$ & 0.435 & 0.586 & 0.568 & 0.398 & 0.412 & 0.520 & 0.629 \\
\hline $\begin{array}{l}\text { Avg. } \\
\text { prec.at } 30 \\
\text { frames }\end{array}$ & 0.423 & 0.528 & 0.514 & 0.368 & 0.405 & 0.468 & 0.553 \\
\hline $\begin{array}{l}\text { Avg. } \\
\text { prec.at 50 } \\
\text { frames }\end{array}$ & 0.410 & 0.489 & 0.469 & 0.324 & 0.369 & 0.434 & 0.494 \\
\hline $\begin{array}{l}\text { Avg. } \\
\text { prec.at } \\
\begin{array}{l}\text { 100 } \\
\text { frames }\end{array}\end{array}$ & 0.354 & 0.396 & 0.412 & 0.310 & 0.342 & 0.397 & 0.421 \\
\hline
\end{tabular}

From Table I, we observe that our combined approach outperforms all the other methods. In short, our experimental results show that by combining various approaches to take advantage of different levels of representations, we achieve better retrieval performance over individual approach. Following figures 8 to figure 13 shows the input, intermediate results and output of proposed content based video retrieval systems. Due to limitation of space we are unable to list java functions and packages used for implementation.

\subsection{Sample Input And Outputs}

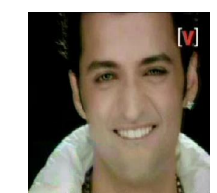

Figure 8. Input Query Image

Algorithm : SimpleColorHistogram

Output : $\min =0, \max =127$

Histogram : RGB 25619401257018481098774552425312231214169176186152174157149

128128125126136118131130110141125134133150138148139134142154163135177168 
The International Journal of Multimedia \& Its Applications (IJMA) Vol.4, No.5, October 2012

180188213231223231215215221227233214231220222239223236236239264255226267 344350381457443446434526512546544530563568575633532552545578547511502521 499465520572588596513597582537490548516520523552562610567592624631601699 695804828929819841729631623490462454431423377393335369393347334409413543 5216235885503563352742021841661581291461361261231171171108792101107115112 133154158137160170154135141154179159157150155127132163149168194204233255 21222521020819516318612415312612313812013992110969595648697819610910498 888979724646364036312626301516161413121361891516711101086675403201 500

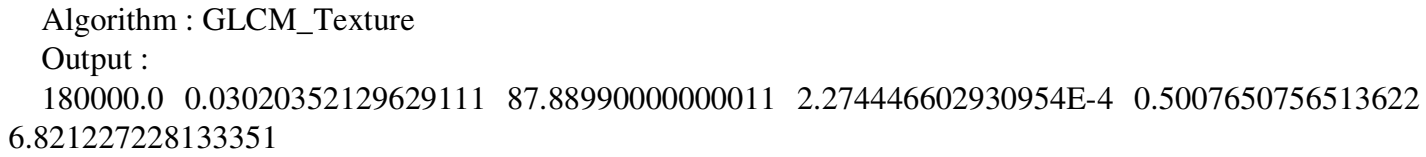

Algorithm : NaiveVector

Output :

NaiveVector java.awt.Color $[\mathrm{r}=0, \mathrm{~g}=0, \mathrm{~b}=0]$ java.awt.Color $[\mathrm{r}=0, \mathrm{~g}=0, \mathrm{~b}=0]$ java.awt.Color $[\mathrm{r}=0, \mathrm{~g}=0, \mathrm{~b}=0]$ java.awt.Color $[\mathrm{r}=0, \mathrm{~g}=2, \mathrm{~b}=1] \quad$ java.awt.Color $[\mathrm{r}=159, \mathrm{~g}=172, \mathrm{~b}=164] \quad$ java.awt.Color $[\mathrm{r}=62, \mathrm{~g}=49, \mathrm{~b}=29]$ java.awt.Color[r=68,g=54,b=33] java.awt.Color[r=111,g=92,b=64] java.awt.Color[r=166,g=179,b=165] java.awt.Color $[\mathrm{r}=119, \mathrm{~g}=125, \mathrm{~b}=113]$

java.awt.Color[ $\mathrm{r}=139, \mathrm{~g}=111, \mathrm{~b}=89]$ java.awt.Color $[\mathrm{r}=183, \mathrm{~g}=151, \mathrm{~b}=135]$ java.awt.Color $[\mathrm{r}=167, \mathrm{~g}=137, \mathrm{~b}=115]$ java.awt.Color $[\mathrm{r}=150, \mathrm{~g}=131, \mathrm{~b}=107]$ java.awt.Color $[\mathrm{r}=132, \mathrm{~g}=113, \mathrm{~b}=80]$ java.awt.Color[r=156,g=124,b=102] java.awt.Color $[\mathrm{r}=75, \mathrm{~g}=61, \mathrm{~b}=36]$ java.awt.Color $[\mathrm{r}=168, \mathrm{~g}=136, \mathrm{~b}=114]$ java.awt.Color $[r=155, g=129, b=110]$ java.awt.Color $[r=125, g=110, b=79]$ java.awt.Color $[r=58, g=32, b=30]$ java.awt.Color $[r=69, g=53, b=38] \quad$ java.awt.Color $[r=66, g=59, b=42]$ java.awt.Color $[r=97, g=107, b=100]$ java. awt. Color $[r=163, g=168, b=152]$ 
The International Journal of Multimedia \& Its Applications (IJMA) Vol.4, No.5, October 2012

\subsection{Screen Shots}

Following figures 9 and 10 demonstartes input and output screens. Due to limitation of space we are unable to show of intermediate results like oracle database, datatables and attribute values, etc..

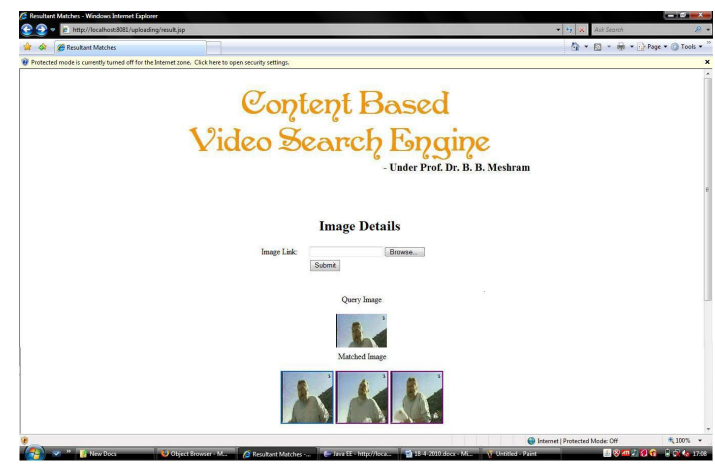

Figure 9. Sreen showing result of match

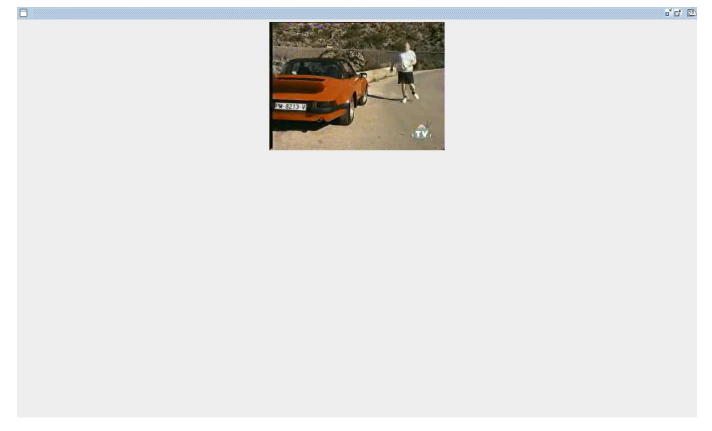

Figure 10. video player maximized

\section{Conclusions}

Video Retrieval is broad area that integrates features from several features and fields including artificial intelligence, machine learning, data base management systems, etc. There have been large numbers of algorithms rooted in these fields to perform various video retrieval tasks. In our proposed system we have implemented retrieval system by integrating various features of query video frame. Experimental results show that integration of extracted features improves video indexing and retrieval. This is demonstrated by the finding that multiple features produce effective and efficient system as precision and recall values are improved. There can be large area of application of proposed system like criminal database retrieval, biomedical information, etc. We further intend to enhance system by integrating more features and other methods and can be extended further to retrieval also from social websites.

\section{REFERENCES}

[1] B. V. Patel, B. B. Meshram, "Content Based Retrieval Systems", International journal of UbiComp, Vol. 3, No. 2, April 2012, pp.13-30.

[2] R Datta, D Joshi, J Li, and J. Wang, (2008) "Image Retrieval: Ideas, Influences, and Trends of the New Age", ACM Computing Surveys, VOl 40, No. 2, April 2008.

[3] B. V. Patel, B. B. Meshram (2007), "Retrieving and Summarizing Images from PDF Documents", International Conference on Soft computing and Intelligent Systems(ICSCSI-07), Jabalpur, India.

[4] Tristan Glatard, Johan Montagnat, Isabelle E. Magnin (2004)," Texture Based Medical Image Indexing and Retrieval: Application to Cardiac Imaging”, MIR'04, October 15-16, 2004, New York, New York.

[5] Egon L. van den Broek, Peter M. F. Kisters, and Louis G. Vuurpijl (2004)," Design Guidelines for a Content-Based Image Retrieval Color-Selection Interface"ACM Dutch Directions in HCI, Amsterdam.

[6] Tristan Glatard, Johan Montagnat, Isabelle E. Magnin (2004), "Texture Based Medical Image Indexing and Retrieval: Application to Cardiac Imaging”, ACM Proc. Of MIR'04, October 15-16, 2004, New York, New York, USA. 
The International Journal of Multimedia \& Its Applications (IJMA) Vol.4, No.5, October 2012

[7] H. Baird, D. Lopresti, B. Davison, W. Pottenger (2004), "Robust document image understanding technologies", HDP, ACM.

[8] R. Datta D. Joshi, J. LI,, JAMES Z. W., "Image Retrieval: Ideas, Influences, and Trends of the New Age”, ACM Transactions on Computing Surveys, Vol. , No. , 20, pp. 1-65.

[9] Jing-Fung Chen,, Hong-Yuan Mark Liao1, and Chia-Wen Lin (2005)," Fast Video Retrieval via the Statistics of Motion Within the Regions-of-Interest", KES'05 Proceedings of the 9th international conference on Knowledge-Based Intelligent Information and Engineering Systems - Volume Part III Springer.

[10] Xi Li, Weiming Hu, Zhongfei Zhang, Xiaoqin Zhang, Guan Luo (2008), “Trajectory-Based Video Retrieval Using Dirichlet Process Mixture Models", 19th British Machine Vision Conference (BMVC).

[11] Lin Lin; Chao Chen; Mei-Ling Shyu; Shu-ChingChen "Weighted Subspace Filtering and Ranking Algorithms for Video Concept Retrieval”, IEEE Multimedia, Volume: 18, Issue: 3, 2011.

[12] Chih-Chin Lai; Ying-Chuan Chen, "A User-Oriented Image Retrieval System Based on Interactive Genetic Algorithm”, IEEE Transactions on Instrumentation and Measurement, Volume: 60 , Issue: 10, 2011.

[13] Shigeaki Sakurai, Hideki Tsutsui, "A clustering method of bloggers based on social annotations", International Journal of Business Intelligence and Data Mining, Volume 6 Issue 1, 2011.

[14] Masami Shishibori, Daichi Koizumi, Kenji Kita, "Fast retrieval algorithm for earth mover's distance using EMD lower bounds and a skipping algorithm”, Advances in Multimedia, Volume 2011

[15] Ballan, L.; Bertini, M.; Del Bimbo, A.; Serra, G, “Video Annotation and Retrieval Using Ontologies and Rule Learning", IEEE Multimedia, Volume: 17, Issue: 4,: 2010.

[16] Beecks, C.; Uysal, M.S.; Seidl, T., “A comparative study of similarity measures for content-based multimedia retrieval”, IEEE International Conference on Multimedia and Expo (ICME), 2010 
Authors

B. V. Patel, is working as Principal at Shah \& Anchor Kutchhi Polytechnic, Mumbai, India. He received his B.E. in Computer Science and Engineering from Karnataka University, India, M.E. in Computer Engineering from VJTI, Mumbai, India. He has authored more than twenty papers in National and International conference and journals. He is also on editorial board of International Journals. His teaching and research interest includes Multimedia Data Mining, Network Management, Network Security, E-learning, etc. He is member of ACM, INENG, IACSIT and CSI.

Dr. B. B. Meshram, is working as Professor \& Head of Computer Technology Dept., VJTI, Matunga, Mumbai. He is Ph.D. in Computer Engineering and has more than 100 papers to his credit at National and International level including international journals. $\mathrm{He}$ is the life member of CSI and Institute of Engineers.
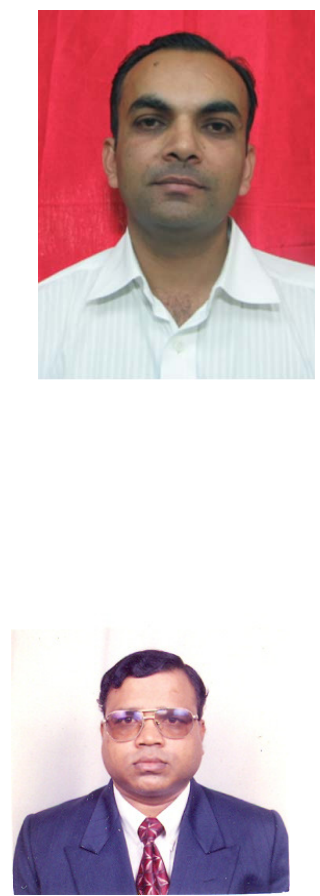